\title{
The Impact of the Oxidative Stress and Inflammatory Process on the Serum Levels of Malonaldialdehyde, Glutathione Peroxidase, and Interlukine-18 in Patients with Essential Hypertension
}

\author{
Salar Fatih Kudhur ${ }^{1}$, Shatha Rouf Moustafa ${ }^{2, *}$ \\ ${ }^{1}$ Ministry of Health, Psychiatric Hospital, Head of Pharmacy Department, Havalan city, Erbil, Iraq, Kurdish \\ ${ }^{2}$ College of Pharmacy, Hawler Medical University, Havalan city, Erbil, Iraq, Arabian
}

Email address:

salarfatih80@yahoo.com (S. F. Kudhur), shatha003@yahoo.com (S. R. Moustafa)

To cite this article:

Salar Fatih Kudhur, Shatha Rouf Moustafa. The Impact of the Oxidative Stress and Inflammatory Process on the Serum Levels of Malonaldialdehyde, Glutathione Peroxidase, and Interlukine-18 in Patients with Essential Hypertension. American Journal of Internal Medicine. Vol. 3, No. 3, 2015, pp. 117-126. doi: 10.11648/j.ajim.20150303.15

\begin{abstract}
Background and objectives: The oxidative stress, antioxidant status and inflammatory process are cooperative events involved in development and progression of essential hypertension. This study was as a step for elucidating the contribution of the malondialdehyde, glutathione peroxidase, interlukine -18 and lipid profile with the incidence, development, and progression of essential hypertension. Aims: The aims of this study were, to assess the change in serum malondialdehyde, glutathione peroxidase, interlukine-18 and lipid profile levels in patients with essential hypertension, moreover, find out the effect of other confounding factors age, gender and stage of the disease on the serum levels of focused parameters and finally detect the correlation between all interested parameters. Patients and Methods: This study was designed to examine the associations between the serum interested parameters levels with the incidence of essential hypertension in 50 patients of both genders, and for the comparing purpose an equal number of the matched age-gender healthy adults also enrolled in this study as a control group. The hypothesis that oxidant /antioxidant status and inflammatory process influence the risk of adverse clinical outcomes are worthy for investigating. Accordingly, malondialdehyde was measured using coloremetric method, while, glutathione peroxidase and interlukine - 18 were measured using enzyme linked immmunosorbent assay and lipid profile was estimated using commercial kit. Results: Patients exhibited a significant elevation in the serum malondialdehyde, glutathione peroxidase, interleukin-18 and lipid profile levels as compared with the control group. Conclusion: The data of the present study indicated that an alteration in oxidant / antioxidant status and inflammatory process in patients with essential hypertension. This investigation provided the first evidence of the ability of malondialdehyde, glutathione peroxidase, interlukine -18 and lipid profile in combination patterns as a factors involved in essential hypertension pathophysiology, etiology and are regarded as a markers of prognostic significance and potential therapeutic targets for future. The demonstration of these parameters provided a new insights into understanding the independence of oxidative stress /antioxidant status and inflammatory pathways in essential hypertension incidence, development and progression.
\end{abstract}

Keywords: Essential Hypertension, Malondialdehyde, Glutathione Peroxidase, Interleukine -18

\section{Introduction}

Essential hypertension (EHT) or hypertension of unknown cause, accounts for more than $90 \%$ of hypertension cases $[1,2]$. Oxidative stress/antioxidant status imbalance and inflammation are considered as significant and novel risk factors for hypertension $[3,4]$. Oxidative stress $(\mathrm{OS})$, which results from either free radical (FR) overproduction or antioxidant exhaustion, has been implicated in the development and progression of hypertension [5].

Evidence has indicated that the OS byproduct malondialdehyde (MDA) increased in patients with EHT [6]. Several studies have involved hypertensive patients and demonstrated that MDA can be a biomarker of oxidative damage [7]. Malondialdehyde is released from the lipoperoxidation of polyunsaturated fatty acids in the cell 
membrane [8]. The exposure to reactive oxygen species (ROS) increases the production of antioxidant enzymes [9].

Glutathione peroxidase (GPX) can be rapidly expressed when cells or organisms are exposed to OS [10]. Chronic low-grade inflammation has been implicated as an integral part of the pathogenesis of vascular diseases [11]. Essential hypertension may contribute to inflammatory diseases [12].

Circulating interleukine-18 (IL-18), as an inflammatory marker, has been found to be a strong predictor of systemic inflammatory processes [13]. Inflammation may be implicated in the development of hypertension, either as a primary or secondary event [14]. The measurement of serum MDA, GPX, IL-18 and lipid profile levels may be considered medically necessary for hypertensive patients concomitant with other risk factors. Limited information is available on the utility of the serum levels of these markers in the assessment of blood pressure in patients with EHT.

The main aim of this study was to determine the levels of oxidant byproduct MDA, antioxidant enzyme GPX, and inflammatory marker IL-18 in patients with EHT. The specific objectives were to assess the lipid profile of essential hypertensive patients as compared with the control group, as well as to assess the association between these parameters and other risk factors in hypertensive patients.

\section{Patients and Methods}

\subsection{Study Design}

This case control study was conducted at the College of Pharmacy, Hawler Medical University, Kurdistan Region, Iraq, from April 2013 to January 2014 and involved 50 patients with EHT and an equal number of matched age -gender apparently healthy adults was also enrolled in this study as a control group for comparing purposes with no history of hypertension or antihypertensive drugs. The participants were selected from the adult patients routinely attending at the Internal Medicine Out Patient Clinic at Erbil Teaching Hospital for follow-up and management. Healthy individuals were selected from the staff and sub-staff of the same hospital. The participants were interviewed and informed about the nature of the study, and all participants provided their verbal consent. The study protocol was approved by the Ethical Committee of the General Director of Health in Erbil Governorate.

Patients with chronic liver disease, renal disease, endocrine dysfunction, and coronary heart disease were excluded from the study. Both groups completed the baseline questionnaire, including the self-reported questions concerning several risk factors for EHT, such as history of diabetes, smoking, physical activity, alcohol consumption, and hormone replacement therapy, as well as anthropometric and hypertension record.

\subsection{Grouping of Patients}

The patient group was classified into stage I and stage II EHT according to the guidelines of Joint National Committee 7 [15] to study the effect of staging on the levels of the focused parameters.

\subsection{Methods}

\subsubsection{Collection of Sample}

Fasting blood samples $(10 \mathrm{~mL})$ were collected from the veins of the participants (healthy adults and patients with EHT) of both genders. The blood samples were left to stand for 30 min to coagulate and then centrifuged for $15 \mathrm{~min}$ at $2500 \mathrm{rpm}$ to $3500 \mathrm{rpm}$. The sera were separated and divided into several portions, after which they were placed into several plastic plain tubes for biochemical tests. The sera were stored at $-20{ }^{\circ} \mathrm{C}$ until analysis (within one to two months). The frozen sera were prepared for measurement by warming at room temperature.

\subsubsection{Biochemical Determinations}

Oxidative stress/antioxidant status imbalance was analyzed through the quantification of MDA via a spectrophotometric method using thiobarbituric and trichlorocetic acids as reagents, MDA reacts with thiobarbituric acid under acidic conditions at $95{ }^{\circ} \mathrm{C}$, forming a pink complex with the maximum absorbance of $532 \mathrm{~nm}$ [16]. The GPX and IL-18 levels were measured using enzyme linked immuno sorbent assay (ELISA). The serum total cholesterol (TC) and high-density lipoprotein-cholesterol (HDLc) triglyceride (TG) levels were determined using commercial enzymatic kits. The low-density lipoprotein-cholesterol (LDLc) level was calculated through Friedewald's formula: LDLc $(\mathrm{mg} / \mathrm{dL})=$ TC (mg/dL) - HDLc (mg/dL) - triglycerides / 5 (mg/dL).

\subsection{Statistical Analyses}

The data were analyzed using the Statistical Patch for Social Sciences v18. The results of the biochemical tests were expressed as mean \pm standard deviation. Furthermore, student t-test was performed to compare two means. Post-hoc test was used to show the significant difference between two of the three variables. Multiple regression was used to reveal the association between each biomarker (as a dependent variable) and several independent variables. $\mathrm{p} \leq 0.05$ was considered statistically significant. The correlations between laboratory findings and continuous variables were evaluated using linear regression analysis.

\section{Results}

This study included 100 individuals (42 men and 58 women) aged between 40 and 65 years. Among the participants, 50 were essential hypertensive patients, who had a mean age of $50.0 \pm 9.4$ years. The remaining 50 participants were apparently healthy adults with mean age of $48.3 \pm 9.6$ years.

\subsection{Effect of Essential Hypertension on the Serum Levels of Focused Parameters}

Hypertensive patients had significantly higher serum MDA, GPX, and IL-18 levels than the control group $\mathrm{p}<0.001$. In addition, the TG, TC, LDLc, and HDLc levels were 
significantly higher in patients than in the control group $\mathrm{p}<$ 0.001 . The mean BMI values were not significantly different (Table 1).

Table 1. The characteristics of the studied participants.

\begin{tabular}{llll}
\hline Parameters & $\begin{array}{l}\text { Patients } \\
\text { N= 50 }\end{array}$ & $\begin{array}{l}\text { Healthy subjects } \\
\mathbf{N = 5 0}\end{array}$ & p- value \\
\hline Age $($ years $)$ & $50.0 \pm 9.4$ & $48.3 \pm 9.6$ & 0.375 \\
SBP $(\mathrm{mmHg})$ & $160 \pm 14$ & $116 \pm 4.6$ & $<0.001$ \\
DBP $(\mathrm{mmHg})$ & $93 \pm 5.4$ & $74 \pm 4.8$ & $<0.001$ \\
MABP $(\mathrm{mmHg})$ & $115 \pm 7.6$ & $88.5 \pm 3.6$ & $<0.001$ \\
BMI $\left(\mathrm{KG} / \mathrm{m}^{2}\right)$ & $23.8 \pm 1.2$ & $24.0 \pm 1.0$ & 0.328 \\
MDA $(\mathrm{nmol} / \mathrm{L})$ & $0.09 \pm 0.027$ & $0.04 \pm 0.02$ & $<0.001$ \\
GPX $(\mathrm{ng} / \mathrm{ml})$ & $1.58 \pm 0.39$ & $1.22 \pm 0.16$ & $<0.001$ \\
IL18 $(\mathrm{pg} / \mathrm{ml})$ & $31.18 \pm 1.27$ & $30.24 \pm 1.09$ & $<0.001$ \\
TG $(\mathrm{mg} / \mathrm{dl})$ & $96.76 \pm 35.1$ & $80.97 \pm 13.2$ & 0.004 \\
TC $(\mathrm{mg} / \mathrm{dl})$ & $175 \pm 32.6$ & $151 \pm 24.4$ & $<0.001$ \\
LDLc $(\mathrm{mg} / \mathrm{dl})$ & $93.8 \pm 30.0$ & $75 \pm 12.29$ & $<0.001$ \\
HDLc $(\mathrm{mg} / \mathrm{dl})$ & $38.5 \pm 4.1$ & $41.5 \pm 5.3$ & 0.002 \\
\hline
\end{tabular}

\subsection{Age Effect}

The effect of age on the levels of the focused parameters in the patient group was shown in (Table 2). In this study, age was classified into four groups (A<40, B 40-49, C 50-59, and
D $60+)$. The results of the statistical analysis showed significant differences among age groups $(<40$ and $60+$, $40-49$ and $60+$, and 50-59 and 60+) in terms of MDA and GPX levels. However, in terms of IL-18 level, significant differences were found between the age groups $<40$ and $60+$, whereas in terms of the TG level, significant differences were found between the age groups 40-49 and 60+and 50-59 and $60+$. In terms of the TC level, significant differences were observed between the age groups 40-49 and 50-59and 40-49 and 60, whereas in terms of LDLc and HDLc levels, significant differences were observed between the age groups $40-49$ and 60+, 40-49 and 60+, and 50-59 and 60+. While the effect of age on the levels of the studied parameters in the control group was shown in (Table 3). The results of the statistical analysis showed a significant differences among the groups ( $<40$ and $50-59,<40$ and $60+, 40-49$ and $60+$, and 40-49 and 50-59) in terms of MDA, GPX, and IL18 levels. Moreover, significant differences were found among age groups ( $<40$ and $50-59$ and $<40$ and $60+$ ) in terms of the TG, TC, and HDLc levels. Meanwhile, no significant differences were found among the age groups in terms of LDLc level.

Table 2. The effects of ages on the mean serum levels of studied parameters in patient group.

\begin{tabular}{|c|c|c|c|c|c|c|}
\hline Parameters & & $\mathrm{N}$ & Mean & $\pm \mathrm{SD}$ & $\mathrm{p}$ & Significance by LSD test \\
\hline \multirow{5}{*}{ MDA } & A- $<40$ & 10 & .08300 & .022201 & \multirow{5}{*}{$<0.001$} & AXD \\
\hline & B- $40-49$ & 10 & .08720 & .013637 & & B X D \\
\hline & C- $50-59$ & 19 & .09042 & .027943 & & C X D \\
\hline & D- $60+$ & 11 & .12464 & .020839 & & \\
\hline & Total & 50 & .09582 & .027323 & & \\
\hline \multirow{5}{*}{ GPX ng/ml } & A- $<40$ & 10 & 1.440 & .0966 & \multirow{5}{*}{0.005} & AXD \\
\hline & B- $40-49$ & 10 & 1.470 & .0949 & & B X D \\
\hline & C- $50-59$ & 19 & 1.511 & .0994 & & C X D \\
\hline & D- $60+$ & 11 & 1.936 & .7284 & & \\
\hline & Total & 50 & 1.582 & .3900 & & \\
\hline \multirow{5}{*}{ IL18pg /ml } & A- $<40$ & 10 & 30.40 & 1.075 & \multirow{5}{*}{0.011} & \\
\hline & B- $40-49$ & 10 & 31.00 & 1.633 & & \\
\hline & C- $50-59$ & 19 & 31.32 & 1.157 & & $\mathrm{AXD}$ \\
\hline & D- $60+$ & 11 & 31.82 & .982 & & \\
\hline & Total & 50 & 31.18 & 1.273 & & \\
\hline \multirow{5}{*}{ TG mg/ dl } & $<40$ & 10 & 96.380 & 43.2412 & \multirow{5}{*}{0.036} & \\
\hline & $40-49$ & 10 & 81.500 & 10.7212 & & \\
\hline & $50-59$ & 19 & 91.658 & 24.4996 & & B X D \\
\hline & $60+$ & 11 & 119.818 & 48.0433 & & C X D \\
\hline & Total & 50 & 96.766 & 35.1154 & & \\
\hline \multirow{5}{*}{ Total cholestrol } & $<40$ & 10 & 164.170 & 27.4153 & \multirow{5}{*}{0.019} & \\
\hline & $40-49$ & 10 & 153.770 & 26.6074 & & B X C \\
\hline & $50-59$ & 19 & 185.116 & 29.3301 & & B X D \\
\hline & $60+$ & 11 & 189.845 & 36.8475 & & \\
\hline & Total & 50 & 175.698 & 32.6459 & & \\
\hline \multirow{5}{*}{ LDLc } & $<40$ & 10 & 73.900 & 18.7169 & \multirow{5}{*}{$<0.001$} & AX D \\
\hline & $40-49$ & 10 & 88.500 & 24.0335 & & B X D \\
\hline & $50-59$ & 19 & 88.847 & 19.8743 & & CX D \\
\hline & $60+$ & 11 & 125.418 & 36.3517 & & \\
\hline & Total & 50 & 93.834 & 30.0949 & & \\
\hline \multirow{5}{*}{ HDLc } & $<40$ & 10 & 40.90 & 3.725 & \multirow{5}{*}{0.02} & AX D \\
\hline & $40-49$ & 10 & 40.70 & 4.084 & & B X D \\
\hline & $50-59$ & 19 & 38.05 & 3.358 & & CX D \\
\hline & $60+$ & 11 & 35.09 & 3.754 & & \\
\hline & Total & 50 & 38.50 & 4.181 & & \\
\hline
\end{tabular}


Table 3. The effects of ages on the mean serum levels of studied parameters in controls groups.

\begin{tabular}{|c|c|c|c|c|c|c|}
\hline Parameters & & $\mathbf{N}$ & Mean & SD & $\mathbf{p}$ & Significance by LSD test \\
\hline \multirow{5}{*}{ MDA } & $A-<40$ & 12 & .0354 & .0126 & & \\
\hline & B- $40-49$ & 10 & .0438 & .0192 & & $\mathrm{AXC}$ \\
\hline & C- $50-59$ & 21 & .0525 & .0194 & 0.006 & AX D \\
\hline & D- $60+$ & 7 & .0654 & .0207 & & $\mathrm{~B} \times \mathrm{C}$ \\
\hline & Total & 50 & .0485 & .0201 & & B X D \\
\hline \multirow{5}{*}{ GPXng/ml } & A- $<40$ & 12 & 1.067 & .1614 & & \\
\hline & B- $40-49$ & 10 & 1.160 & .1506 & & $\mathrm{AXC}$ \\
\hline & C- $50-59$ & 21 & 1.295 & .1117 & $<0.001$ & $\mathrm{AXD}$ \\
\hline & D- $60+$ & 7 & 1.343 & .0787 & & $\mathrm{~B} \times \mathrm{C}$ \\
\hline & Total & 50 & 1.220 & .1641 & & B X D \\
\hline \multirow{5}{*}{ IL18 pg/ml } & A- $<40$ & 12 & 29.58 & .793 & & \\
\hline & B- $40-49$ & 10 & 29.80 & 1.229 & 0.001 & $\mathrm{AXC}$ \\
\hline & C- $50-59$ & 21 & 30.62 & 1.024 & & $\mathrm{AXD}$ \\
\hline & D- $60+$ & 7 & 30.86 & .900 & & $\mathrm{~B} \times \mathrm{C}$ \\
\hline & Total & 50 & 30.24 & 1.098 & & B X D \\
\hline \multirow{5}{*}{$\mathrm{TG} \mathrm{mg} / \mathrm{dl}$} & $<40$ & 12 & 72.100 & 12.4213 & & \\
\hline & $40-49$ & 10 & 81.750 & 8.7631 & & $\mathrm{AXC}$ \\
\hline & $50-59$ & 21 & 83.295 & 13.1628 & 0.04 & $\mathrm{AXD}$ \\
\hline & $60+$ & 7 & 88.114 & 15.2260 & & \\
\hline & Total & 50 & 80.974 & 13.2984 & & \\
\hline \multirow{5}{*}{ Total cholesterol mg/dl } & $<40$ & 12 & 135.808 & 9.821 & & \\
\hline & $40-49$ & 10 & 151.360 & 28.135 & 0.05 & $\mathrm{AXC}$ \\
\hline & $50-59$ & 21 & 155.105 & 26.135 & & AXD \\
\hline & $60+$ & 7 & 165.286 & 22.171 & & \\
\hline & Total & 50 & 151.150 & 24.473 & & \\
\hline \multirow{5}{*}{ LDLc mg/dl } & $<40$ & 12 & 70.425 & 13.853 & & \\
\hline & $40-49$ & 10 & 72.300 & 11.106 & & \\
\hline & $50-59$ & 21 & 76.829 & 10.820 & 0.194 & Non Sig. \\
\hline & $60+$ & 7 & 81.771 & 13.712 & & \\
\hline & Total & 50 & 75.078 & 12.2941 & & \\
\hline \multirow{5}{*}{ HDLc mg/dl } & $<40$ & 12 & 45.42 & 5.838 & & \\
\hline & $40-49$ & 10 & 41.40 & 6.059 & 0.019 & $\mathrm{AXC}$ \\
\hline & $50-59$ & 21 & 40.24 & 4.206 & & AX D \\
\hline & $60+$ & 7 & 38.71 & 3.498 & & \\
\hline & Total & 50 & 41.50 & 5.354 & & \\
\hline 3.3. Gender Effect & & & & HDLc (mg/dl) & $37.19 \pm 3.01$ & $39.45 \pm 3.43$ \\
\hline
\end{tabular}

The effect of gender on the levels of the focused parameters in the patient group was presented in (Table 4). Men and women exhibited a significant difference in serum MDA levels $\mathrm{p}$ value $<0.05$, GPX $\mathrm{p}=0.048$, LDLc $\mathrm{p}<0.001$, HDLc $\mathrm{p}=$ 0.059 other parameters, while, in case of IL-18 there was a difference ,but this difference reach near the significant level $p=0.064$. While, the effect of gender on the levels of the focused parameters in the control group was shown in (Table 5). There were significant differences between men and women in term of all parameters except for age and BMI.

Table 4. The effect of gender on the serum levels of the studied parameters in patient group.

\begin{tabular}{llll}
\hline Parameters & Men $\mathbf{N}=\mathbf{2 1}$ & Women N=29 & p- value \\
\hline Age $($ years $)$ & $50.0 \pm 9.4$ & $48.3 \pm 9.6$ & 0.128 \\
SBP $(\mathrm{mmHg})$ & $160 \pm 14$ & $116 \pm 4.6$ & 0.924 \\
DBP $(\mathrm{mmHg})$ & $93 \pm 5.4$ & $74 \pm 4.8$ & 0.787 \\
MABP $(\mathrm{mmHg})$ & $115 \pm 7.6$ & $88.5 \pm 3.6$ & 0.967 \\
BMI $\left(\mathrm{KG} / \mathrm{m}^{2}\right)$ & $23.8 \pm 1.2$ & $24.0 \pm 1.0$ & 0.338 \\
MDA $(\mathrm{nmol} / \mathrm{L})$ & $0.1 \pm 0.26$ & $0.08 \pm 0.02$ & 0.031 \\
GPX $(\mathrm{ng} / \mathrm{ml})$ & $1.71 \pm 0.57$ & $1.49 \pm 0.11$ & 0.048 \\
IL18 $(\mathrm{pg} / \mathrm{ml})$ & $31.57 \pm 1.43$ & $30.9 \pm 1.08$ & 0.064 \\
TG $(\mathrm{mg} / \mathrm{dl})$ & $104.38 \pm 43.19$ & $91.25 \pm 27.39$ & 0.195 \\
TC $(\mathrm{mg} / \mathrm{dl})$ & $184.27 \pm 32.38$ & $169.47 \pm 31.95$ & 0.11 \\
LDLc $(\mathrm{mg} / \mathrm{dl})$ & $116.79 \pm 30.96$ & $77.2 \pm 14.77$ & $<0.001$ \\
\hline
\end{tabular}

Table 5. The effect of gender on the serum levels of the studied parameters in controls group.

\begin{tabular}{llll}
\hline Parameters & Men N=21 & Women N=29 & p- value \\
\hline Age $(\mathrm{years})$ & $50.0 \pm 9.4$ & $48.3 \pm 9.6$ & 0.375 \\
SBP $(\mathrm{mmHg})$ & $160 \pm 14$ & $116 \pm 4.6$ & $<0.001$ \\
DBP $(\mathrm{mmHg})$ & $93 \pm 5.4$ & $74 \pm 4.8$ & $<0.001$ \\
MABP $(\mathrm{mmHg})$ & $115 \pm 7.6$ & $88.5 \pm 3.6$ & $<0.001$ \\
BMI $\left(\mathrm{KG} / \mathrm{m}^{2}\right)$ & $23.8 \pm 1.2$ & $24.0 \pm 1.0$ & 0.328 \\
MDA $(\mathrm{nmol} / \mathrm{L})$ & $0.06 \pm 0.01$ & $0.03 \pm 0.01$ & $<0.001$ \\
GPX $(\mathrm{ng} / \mathrm{ml})$ & $1.30 \pm 0.14$ & $1.15 \pm 0.15$ & 0.001 \\
IL18 $(\mathrm{pg} / \mathrm{ml})$ & $31.05 \pm 0.95$ & $29.66 \pm 0.76$ & $<0.001$ \\
TG $(\mathrm{mg} / \mathrm{dl})$ & $85.88 \pm 13.01$ & $77.47 \pm 12.58$ & 0.027 \\
TC $(\mathrm{mg} / \mathrm{dl})$ & $167.53 \pm 19.99$ & $139.28 \pm 20.38$ & $<0.001$ \\
LDLc $(\mathrm{mg} / \mathrm{dl})$ & $80.83 \pm 12.11$ & $70.9 \pm 10.8$ & 0.004 \\
HDLc $(\mathrm{mg} / \mathrm{dl})$ & $38.67 \pm 3.2$ & $43.55 \pm 3.43$ & 0.001 \\
\hline
\end{tabular}

\subsection{Staging Effect}

The serum MDA level was significantly higher $\mathrm{p}$ value $<$ 0.05 in stage II hypertensive patients than in stage I. Overall, the levels of the other parameters were not significantly different, except for TG and HDLc $\mathrm{p}=0.013, \mathrm{p}=<0.001$ respectively (Table 6). 
Table 6. Comparison between stage I and stage II hypertensive patients regarding the serum levels of studied parameters.

\begin{tabular}{llll}
\hline Parameters & $\begin{array}{l}\text { Stage I } \\
\mathbf{N = 2 2}\end{array}$ & $\begin{array}{l}\text { Stage II } \\
\mathbf{N = 2 8}\end{array}$ & p- value \\
\hline Age (years) & $50.0 \pm 9.4$ & $48.3 \pm 9.6$ & 0.375 \\
SBP $(\mathrm{mmHg})$ & $160 \pm 14$ & $116 \pm 4.6$ & $<0.001$ \\
DBP $(\mathrm{mmHg})$ & $93 \pm 5.4$ & $74 \pm 4.8$ & $<0.001$ \\
MABP $(\mathrm{mmHg})$ & $115 \pm 7.6$ & $88.5 \pm 3.6$ & $<0.001$ \\
BMI $\left(\mathrm{KG} / \mathrm{m}^{2}\right)$ & $23.8 \pm 1.2$ & $24.0 \pm 1.0$ & 0.328 \\
MDA $(\mathrm{nmol} / \mathrm{L})$ & $0.08 \pm 0.02$ & $0.10 \pm 0.02$ & 0.014 \\
GPX $(\mathrm{ng} / \mathrm{ml})$ & $1.47 \pm 0.12$ & $1.66 \pm 0.49$ & 0.07 \\
IL18 $(\mathrm{pg} / \mathrm{ml})$ & $30.64 \pm 0.95$ & $31.61 \pm 1.34$ & 0.06 \\
TG $(\mathrm{mg} / \mathrm{dl})$ & $83.07 \pm 22.72$ & $107.19 \pm 39.5$ & 0.013 \\
TC $(\mathrm{mg} / \mathrm{dl})$ & $172 \pm 39.54$ & $178 \pm 32.0$ & 0.5 \\
LDLc $(\mathrm{mg} / \mathrm{dl})$ & $84.9 \pm 17.87$ & $100.8 \pm 35.7$ & 0.062 \\
HDLc $(\mathrm{mg} / \mathrm{dl})$ & $41.36 \pm 3.2$ & $36.25 \pm 3.43$ & $<0.001$ \\
\hline
\end{tabular}

\subsection{Correlation Coefficient}

The correlation between systolic and diastolic blood pressures with the focused parameters were represented in (Tables 7,8).

Table 7. Relationship between systolic blood pressure and the parameters in hypertensive patients.

\begin{tabular}{lll}
\hline parameters & r-value & p-value \\
\hline MDA & 0.68 & $<0.001$ \\
GPX & 0.65 & $<0.001$ \\
IL-18 & 0.63 & $<0.001$ \\
TG & 0.39 & $<0.001$ \\
TC & 0.51 & $<0.001$ \\
LDLc & 0.61 & $<0.001$ \\
HDLc & -0.057 & $<0.001$ \\
\hline
\end{tabular}

Table 8. Relationship between diastolic blood pressure and the parameters in hypertensive patients.

\begin{tabular}{lll}
\hline parameters & r-value & p-value \\
\hline MDA & 0.68 & $<0.001$ \\
GPX & 0.65 & $<0.001$ \\
IL-18 & 0.63 & $<0.001$ \\
TG & 0.39 & $<0.001$ \\
TC & 0.51 & $<0.001$ \\
LDLc & 0.61 & $<0.001$ \\
HDLc & -0.057 & $<0.001$ \\
\hline
\end{tabular}

\section{Discussion}

\subsection{General View}

Oxidative stress and inflammation are considered as a significant and novel risk factors for coronary diseases, such as hypertension $[3,4]$. The actual functions of these processes in the development and progression of EHT remain unclear.

\subsection{The Effect of Essential Hypertension on the Serum Levels of Studied Parameters}

\subsubsection{The Effect of Essential Hypertension on the Serum Levels of Malondialdehyde}

This study revealed that the OS biomarker MDA level was significantly higher in patients with EHT than in the control group $\mathrm{p}<0.001$ (Table 1).

This finding is supported by the results of previous studies that reported increased MDA levels in patients with EHT. Therefore, the result of the present study was agreeable with that of previous studies [17-24]. Accordingly, Os has been implicated in EHT pathogenesis.

\subsubsection{The Effect of Essential Hypertension on the Serum Levels of Glutathione Peroxidase}

The results of the statistical analysis revealed a significant increase in GPX levels in patients with EHT as compared with the control group $\mathrm{p}<0.001$ (Table 1). This result was concordant with the findings of previous studies [25-27], in which a significant increase in the GPX levels was reported. It was published that the GPX levels in prehypertensive individuals significantly increased as compared with that in control individuals [28]. This result may be partially explained by the increased OS caused by the overproduction of ROS radicals in patients with EHT and is paralleled by a significant increase in the levels of relevant enzymes, particularly GPX. Glutathione peroxidase has been observed to be rapidly expressed when organisms or cells are exposed to OS [29]. Several researchers have reported contradictory findings on the antioxidant status of patients with EHT. The result of the present study was not consistent with the findings of previous studies [19,20,23,30-32]. These contradicting findings might be explained by the recent diagnosis of patients in the current study and by the absence of continuous exposure to hydrogen peroxide, hydrogen peroxynitrite, and other free radicals to overwhelm the activity of the antioxidant enzyme GPX. This inconsistency might generally be understood on the basis of methodological variations. Data may be obtained from other demographic groups, thus giving rise to variations related to patient lifestyles.

\subsubsection{The Effect of Essential Hypertension on the Serum Levels of Interlukine-18}

The present study revealed a significant increase in the serum IL-18 level as compared with the control group $\mathrm{p}<$ 0.001 (Table1). Elevated serum IL-18 levels have been shown to be a predictive parameter for the development and progression of EHT. The result of the present study was concordant with the finding of previous study [33]. Interlukine -18 is apparently an attractive candidate biomarker for the diagnosis and prognosis of patients with EHT. Indeed, IL-18 provides a significant prognostic information as opposed to the classic lipid profile and inflammatory markers [34]. In this study, IL-18 was evaluated as a serum marker for inflammation and has been evaluated as a potential tool for EHT risk prediction. Thus far, the presented data support the association between inflammation and EHT incidence. The data also support the hypothesis suggesting that the IL-18 levels might serve a key function in inflammatory response, which contributes to the EHT incidence. Therefore, IL-18 is pro-inflammatory cytokine possibly implicated in EHT pathogenesis.

\subsubsection{The Effect of Essential Hypertension on the Serum Levels of Lipid Profile}

The results of the statistical analysis revealed that highly 
significant differences existed in the TG, TC, LDLc, and HDLc levels in patients with EHTas compared with the control group $\mathrm{p}=0.004,0.001,0.001$, and 0.002 , respectively (Table 1). Essential hypertension was associated with lipoperoxidation, and an imbalance in the antioxidant status suggested that OS is important in EHT pathogenesis.

\subsection{Age Effect}

The effects of age on the levels of the focused parameters in the patient group was shown in (Table 2). The results of the statistical analysis showed that significant differences existed between age groups $<40$ and $60+, 40-49$ and $60+$, and 50-59 and $60+$ in terms of MDA and GPX levels. In terms of IL-18 level, significant differences were found between the age groups $<40$ and 60+, whereas in terms of TG levels, significant differences were found between the age groups 40-49 and 60+and 50-59 and 60+. In terms of TC levels, significant differences were found between the age groups 40-49 and 50-59and 40-49 and 60+. Finally, in terms of LDLc and HDLc levels, significant differences were found between the age groups 40-49 and 60+, 40-49 and 60+, and 50-59 and $60+$.

In the control group, the results of the statistical analysis showed significant differences between the age groups $<40$ and 50-59, $<40$ and $60+, 40-49$ and $60+$, and 40-49 and $50=59$ in terms of the MDA, GPX and IL1 8 levels. Moreover, significant differences were observed between the age groups $<40$ and 50-59and $<40$ and $60+$ in terms of TG, TC, and HDLc levels. Meanwhile, no significant difference was observed between the age groups in terms of LDLc level (Table 3).

The result of the current study was agreeable with the concept that advanced age is considered as a risk factor for getting EHT, the mean age at diagnosis was $50.0 \pm 9.4$ (Table 1 ), so this finding was in harmony with [35] who published that, the most important risk factor for hypertension in large populations is the age effect.

This study was designed to investigate the age effect on the relationship between oxidative stress/anti-oxidative status and the inflammatory process with EHT. Hypertension can also be age-related, it is not a chronic disease, but it is independently associated with cardiovascular diseases in the elderly, which is why aging and hypertension are well-documented cardiovascular risk factors [36]. Most structural vascular and function alterations result in cardiovascular complications, such as in aging and hypertension [37,38]. Moreover, the vascular changes associated with EHT are generally considered to be related to the aging process [39]. The increased prevalence of hypertension with advanced age [40] was also evident. Blood pressure tends to increase with age, such that a higher prevalence of hypertension could be expected as a consequence of the growing elderly population [41]. In healthy individuals and hypertensive patients, increasing age was associated with the progressive and specific decrease in vasodilation related to acetylcholine. These results support the concept suggesting that advancing age is an independent factor leading to the progressive impairment of endothelium-dependent vasodilation in humans $[42,43]$. They were shown that GPX level was significantly higher $\mathrm{P}<0.05$ in elderly hypertensive patients than in the control group [44,45]. These results were consistent with those of the present study (Table 2). The observed GPX activities were contradictory, and whether GPX activity decreases [46] or increases [47] with age remains unclear. Moreover, no alteration in the GPX level was observed with aging $[48,49]$. Studies have already established that aging is associated with a disruption of glutathione metabolism [50,51]. The decrease in GPX activity with age could be caused by selenium deficiency, which may be associated with the poor diet of elderly people or with the oxidative modifications in enzymatic proteins. Researchers have emphasized that ROS-induced protein damage may be associated with increasing age [52]. It was reported the function of the variations in IL-18 level and function in elderly people [53]. Interlukine-18 levels are related to physical function in 65- to 80 -year old individuals. Interlukine -18 may serve an important function in age-related functional impairment.

\subsection{Gender Effect}

The serum GPX levels in women with EHT were significantly lower than those in men (Table 4). In other study, no difference in enzyme activity was observed between men and women with EHT [54]. In addition, (Table 4) shows no significant difference between men and women with EHT in terms of IL-18 level $p=0.064$. By contrast, [55] reported that women with EHT exhibited reduced IL-18 levels because of the small population size or because of methodological, demographical and life style variations. Studies reported that the prevalence of hypertension is higher in men than in women at younger ages [56]. This finding can be attributed to the fact that women are more protected from OS because of estrogen effect [57]. Thus, the differences in the susceptibility to OS between men and women have been emphasized. They were reported that the level of oxidative damage in the DNA is higher in men than in women [58,59]. Other studies demonstrated that oxidative damage to mitochondrial DNA is significantly lower in female rats than in male rats $[60,61]$. Although numerous studies have been conducted on the oxidant-antioxidant imbalance in hypertension, supporting data for men at the early stages of hypertension remain limited.

The healthy men had higher MDA, GPX, and IL-18 levels, as well as lipid profile $\mathrm{p}<0.05$, than the healthy women (Table 5 ). The findings of the present study were concordant with those of previous studies [35], which reported that GPX activity decreased in women with similar ages and from a healthy population compared with healthy men.

\subsection{Staging Effect}

There were a significant differences between stages I and II in terms of the MDA level $\mathrm{p}=0.05$ (Table 6). Meanwhile, it was reported that MDA levels significantly increased in the stage I and II hypertension groups as compared with the 
control group $\mathrm{p}<0.05$ [23].

No significant differences were observed between stages I and II in terms of the GPX and IL-18 levels $\mathrm{p}=0.07$ and 0.06 respectively (Table 6). These findings might be attributed to the small number of participants.

The activity of the Se-dependent enzyme GPX has been reported to increase in patients with different stages of EHT $[62,63]$. By contrast, it was demonstrated that GPX level significantly decreased $\mathrm{p}<0.05$ in the prehypertension, stage I, and stage II hypertension groups compared with the control group [23].

It was hypothesized that the body tends to combat stress through the overexpression of the GPX gene, which serves as the first line of defense in EHT. As the severity of hypertension advances to stages I and II, even the defensive mechanism via GPX may deteriorate because of the enhanced production of free radicals, which may be the reason for the reduced GPX levels [64].

In terms of IL-18 level, previous studies were carefully reviewed and detected that no results that supported this finding were found. Thus, no data concerning the effect of staging level on serum IL-18 level were present. Accordingly, the present study is the first attempt to investigate the effect of staging on the IL-18 levels in patients with EHT.

\subsection{Correlation Coefficient}

Tables 7 and 8 show the association between the focused parameters and hypertension were shown in (Tables 7,8). Moderately positive correlations were observed among MDA, GPX, and IL-18 levels, as well as the lipid profile in hypertensive patients, except for HDLc, which had moderately negative correlation. This finding demonstrated a moderately linear correlation between the studied parameters and hypertension. The results of the present study were consistent with those of previous studies [55], in which IL-18 level has been reported to be positively correlated with TGs but negatively correlated with HDLc. Previous studies were carefully reviewed, but no previous data were found to support this finding. A positive correlation was found between oxidative stress and blood pressure by measuring MDA as a marker of oxidative stress, this finding was concordant with the results of a previous study [23].

Clinical Implications: The present study supported the increasing evidence suggesting that the MDA, GPX, and IL-18 levels, as well as the lipid profile, may serve as alternative markers for the clinical evaluation and management of EHT. According to the results of the previous studies and of the present study, OS may be considered as a novel therapeutic target for EHT. This study speculated that inflammation mediated by the elevated serum IL-18 level represents a mechanism that accelerates the development of EHT. Thus, the clinical implication of the present study is that the suppression or antagonism of IL-18 might be clinically beneficial.

In this work, the potential function of OS in the development and progression of EHT was examined by estimating the MDA level as a marker of lipid peroxidation. In addition, the relationship of oxidant/antioxidant balance and inflammatory process with EHT incidence was investigated. For this purpose, the serum MDA level was measured as an index of lipids and as a marker of OS, whereas serum GPX activity was determined as an antioxidant enzyme, and the IL-18 level was investigated as an inflammatory marker. Enhanced OS mediates the endothelial dysfunction associated with hypertension. The aim of the present study was to investigate the relative contributions of the oxidant/anti-oxidant enzymes and inflammatory processes to the pathogenesis of endothelial dysfunction in EHT. Increasing evidence of the importance of ROS highlights the need for reliable and reproducible markers of oxidative stress, the assessment of which can be used to monitor treatment-induced changes. Given the relationship between OS and hypertension, drugs with antioxidant effects can be expected to lower the BP. The inflammatory state in hypertension may provide a new therapeutic target for future drug design. Thus, the inhibition of IL-18 may provide a new therapeutic strategy for EHT. The pharmacologic targeting of IL-18 may supply an effective strategy to control EHT. The observed increase in inflammatory parameters in subjects who subsequently developed EHT is particularly relevant and confers options for potential primary prevention strategies.

\section{Conclusion}

Compared with healthy participants, patients with EHT exhibited higher serum MDA, GPX, IL-18 levels and lipid profile. The coexistence of OS and inflammation with EHT was significantly correlated with serum MDA $r=0.68, p$ $<0.05$ and IL-18 $r=0.63, p<0.05$ levels. The progression of hypertension (stage II) demonstrated by increased blood pressure was associated with increased serum MDA, GPX, and IL-18 levels.

Further elaborated studies including larger sample size are needed to verify the role of oxidative stress / antioxidant status and proinflammatory cytokine activation in essential hypertension. Further studies are warranted in order to assess their utility as a predictors of the presence of essential hypertension.

\section{Abbreviations}

BMI: Body mass index, BP: Blood pressure, DBP: Diastolic blood pressure, ELISA: Enzyme linked immuno sorbent assay, EHT: Essential hypertension, FR: Free radical, GPX: Glutathione peroxidase, HDLc: High density lipoprotein, IL-18: Interlukine-18, LDLc: Low density lipoprotein, MRBP: Mean arterial blood pressure, MDA: Malondialdehyde, OS: Oxidative stress, ROS: Reactive oxygen species, SBP: Systolic blood pressure, Se :Selenium, SOD: Superoxide dismutase, TC: Total cholesterol, TG: Triglyceride. 


\section{Author' Contributions:}

All authors read and approved the final manuscript.

\section{Financial Support}

This article was partially supported by Hawler Medical University.

This article is M.Sc. thesis which was performed under my supervision at the Department of Clinical Analysis, College of Pharmacy, Hawler Medical University for Degree in M.Sc. of Science in Clinical Biochemistry .

\section{References}

[1] Lifton RP., Gharavi AG., Geller DS. (2001). Molecular mechanisms of human hypertension. Cell, 104: 545- 556.

[2] Johnson R.J., Herrera-Acosta J., Schreiner G.F., Rodriguez-Iturbe B. (2002). Subtle acquired renal injury as a mechanism of salt-sensitive hypertension. N Engl J Med, 346: 913-923.

[3] Kotur-Stevuljevic J., Memon L., Stefanovic A. (2007). Correlation of oxidative stress parameters and inflammatory markers in coronary artery disease patients. Clin Biochem, 40: 181-187.

[4] Soydinç S, Çelik A, Demiryürek S. (2007). The relationship between oxidative stress, nitric oxide, and coronary artery disease. Eur J Gen Med, 4(2): 62- 66.

[5] Ogita H., Liao J. (2004). Endothelial function and oxidative stress. Endothelium, 11: 123- 132.

[6] Paravicini .TM. , Touyz, R.M. (2006). Redox signaling in hypertension. Cardiovasc. Res, 71: 247-258.

[7] Kędziora-Kornatowska K., Szewczyk-Golec K., Czuczejko J. (2007). Effect of melatonin on the oxidative stress in erythrocytes of healthy young and elderly subjects. J Pineal Res, 42: $153-158$.

[8] Das SK, Sanyal K, Basu A. (2005). Study of urban community survey in India: Growing trend of high prevalence in developing country. Int J Med Sci, 2: 70-78.

[9] Wilcox C.S., Pearlman A. (2008). Chemistry and antihypertensive effects of tempol and other nitroxides. Pharmacol Rev, 60: 418-469.

[10] Pompella A., Visvikis A., Paolicchi A., De Tata V., Casini A.F. (2003). The changing faces of glutathione, a cellular protagonist. Biochemical Pharmacology, 66(8): 1499-1503.

[11] Engstrom G., Janson L., Berglund G. (2003). Inflammation-sensitive plasma proteins and incidence of myocardial infarction in men with low cardiovascular risk. Arterioscler Thromb Vasc Biol, 23: 2247- 2251.

[12] Pedrinelli R. (2000). Endothelial vasomotor dysfunction in hypertension. J Hum Hypertens, 14: 481- 483.

[13] Bautista LE, Vera L.M., Arenas I.A., Gamarra G. (2005). Independent association between inflammatory markers (C-reactive protein, interleukin- 6, and TNF-alpha) and essential hypertension. J Hum Hypertens, 19: 149- 154.
[14] Nagano M., Nakamura M., Sato K., Tanaka F., Segawa T., Hiramori K. (2005). Association between serum C-reactive protein levels and pulse wave velocity. a population-based cross-sectional study in a general population. Atherosclerosis, 180: $189-195$.

[15] Chobanian A.V., Bakris G. L., Black H. R. (2003). The seventh report of the joint national committee on prevention, detection, evaluation, and treatment of high blood pressure: the JNC 7 report. The Journal of the American Medical Association, 289 (19): $2560-2572$.

[16] Lee R., Margaritis M., Channon K.M., Antoniades C. (2012). Evaluating oxidative stress in human cardiovascular disease: methodological aspects and considerations. Curr Med Chem, 19(16): 2504- 2520 .

[17] Babu Raja Maharjan, Jha J.C., Vishwanath P., Alurkar V.M., Singh P.P. (2008). Oxidant-antioxidant Status and Lipid Profile in the Hypertensive Patients. Journal of Nepal Health Research Council, 6 (2): 63- 68.

[18] Khanna H. D., Sinha M. K., Khanna S., Tandon R. (2008). Oxidative Stress in hypertension: Association with antihypertensive treatment. Indian J Physiol Pharmacol, 52(3): $283-287$.

[19] Seema L., Jaw alekar, Ujjwala J., Kulkarni, Vasant T., Surve and Deshmukh Y. A. (2010). Role of Oxidants and Anti Oxidants in Patients with Cardiovascular Diseases. Asian J. Med. Sci, 2(4): 181- 184.

[20] Kornelia Kędziora-Kornatowska, Jolanta Czuczejko, Jadwiga Motyl, Karolina Szewczyk-Golec, Mariusz Kozakiewicz, Hanna Pawluk, Józef Kędziora, Robert Błaszczak, Maciej Banach,Jacek Rysz (2010). Effects of coenzyme Q10 supplementation on activities of selected antioxidative enzymes and lipid peroxidation in hypertensive patients treated with indapamide. A pilot study.Arch Med Sci, 6(4): 513- 518.

[21] Meera K.S. (2011). Oxidative imbalance in smokers with and without hypertension. Biomedical Research, 22 (3): 267- 272.

[22] Shamil H., Othman, Imad A., Thanoon, (2012). Oxidant / antioxidant status and thyroid function tests in hypertensive patients treated by captopril or valsartan. Ann. Coll. Med. Mosul, 38(2): 62 - 67.

[23] Aquil A., Mohd M.H., Usha S., Najmul I. (2013). Comparative study of marker of oxidative stress among normotensive, pre-hypertensive and hypertensive subjects. Biomedical Research, 24 (4): 493- 497.

[24] Komala PT, Sankar P, Malliga S. (2013). Comparative Study of Antihypertensive Treatment with Enalapril and Atenolol on Oxidative Stress. JPBS, 5(4): 8-11.

[25] Nandeesha H., Sathiyapriya V., Bobby Z., Pavithran P., Agrawal A.,Selvaraj N. (2007). Altered Oxidant-Antioxidant Status in Non-Obese Men with Moderate Essential Hypertension. Indian J Med Sci, 61: 326- 331.

[26] Selvaraj N., Sathiyapriya V., Bobby Z., Nandeesha H., Aparna A. (2013). Elevated glutathione peroxidase in newly diagnosed hypertension: its relation to insulin resistance. Clin Exp Hypertens, 35 (3): 195- 199.

[27] Nambiar S., Viswanathan S., Zachariah B., Hanumanthappa N., Agrawal A. (2013). Elevated Glutathione Peroxidase in Newly Diagnosed Hypertension: Its Relation to Insulin Resistance. Clinical and Experimental Hypertension, 35 (3): 195- 199. 
[28] Sathiyapriya V., Nandeesh H., Bobb Z., Selvaraj N., Pavithran P. (2007). Perturbation of oxidant-antioxidant status in non-obese prehypertensive male subjects. Journal of Human Hypertension, 21: $176-178$

[29] Lu D., Maulik N., Moraru I.I., Kreutzer D.L., Das D.K. (1993). Molecular adaptation of vascular endothelial cells to oxidative stress. Am J Physiol, 264: 715- 722.

[30] Al-Solaiman Y., Jesri A., Zhao Y., Morrow J.D., Egan B.M. (2009). Low-Sodium DASH reduces oxidative stress and improves vascular function in salt-sensitive humans. J Hum Hypertension, 23: 826- 835.

[31] Rybka J. , Kupczyk D. , dziora-Kornatowska K., Ke, Motyl J. , Czuczejko J. , Szewczyk-Golec K. (2011). Glutathione-Related Antioxidant Defense System in Elderly Patients Treated for Hypertension. Cardiovasc Toxicol, 11: 1-9.

[32] Rodrigo R., Gonzalez J., Paoletto F. (2011). The role of oxidative stress in the pathophysiology of hypertension. Hypertens Res, 34: 431- 440.

[33] Evans J., Collins M., Jennings C., van der Merwe L., Söderström I., Olsson T., Levitt N.S., Lambert E.V., Goedecke J.H. (2007). The association of interleukin-18 genotype and serum levels with metabolic risk factors for cardiovascular disease. Eur J Endocrinol. 157(5): 633- 640.

[34] Stefan Blankenberg, Gérald Luc, Pierre Ducimetière, Dominique Arveiler, Jean Ferrières, Philippe Amouyel, Alun Evans, François Cambien , Laurence Tiret, (2003). Interleukin-18 and the Risk of Coronary Heart Disease in European Men. Circulation, 108: 2453- 2459.

[35] Lewington S., Clarke R., Qizilbash N., Peto R., Collins R. (2002). Age specific relevance of usual blood pressure to vascular mortality: a meta-analysis of individual data for one million adults in 61 prospective studies. Lancet, 360: 190321913

[36] Roberts W.C. (1980). The hypertensive diseases. In Laragh JH, ed. Topics in Hypertension. New York: York Medical Books, 17: $368-388$

[37] Lüscher T.F., Vanhoutte P.M. (1990). The Endothelium: Modulator of Cardiovascular Function. Boca Raton, Fla: CRC Press Inc, 1-228.

[38] Ross R. (1993). The pathogeneses of atherosclerosis: an update. N Engl J Med, 314: 488- 500.

[39] Soltis E.E. (1987). Effect of age on blood pressure and membrane dependent vascular responses in the rat. Circ Res, 61 889- 897 .

[40] Tassaduqe K., Ali M., Salam A., Latif M. (2004). Hypertension in Relation to Obesity, Smoking, Stress, Family History, Age and Marital Status among Human Population of Multan. Pak J Med Sci, 35: 30- 35

[41] Levenson J.W., Skerrett P.J., Gaziano J.M. (2002). Reducing the global burden of cardiovascular disease: the role of risk factors. Prev Cardiol, 5: 188- 199.

[42] Gerhard M., Roddy M.A., Creager S.J., Creager M.A. (1996). Aging progressively impairs endothelium-dependent vasodilation in forearm resistance vessels of human. Hypertension, 27: 849-853.

[43] Taddei S, Ghiadoni L, Virdis A, Versari D, Salvetti A. (2003).
Mechanisms of endothelial dysfunction: clinical significance and preventive non-pharmacological therapeutic strategies. Curr Pharm, 9: 2385- 2402.

[44] Looi M., Noor A. A., Yasmin A. M. (2005). Effects of Palmvitee on Status of Superoxide Dismutase and Glutathione Peroxidase in Rat Liver during Aging. Malaysian Journal of Biochemistry and Molecular Biology, 12: 21- 24.

[45] Rybka J., Kupczyk D., Kędziora-Kornatowska K., Pawluk H., Czuczejko J., Szewczyk-Golec K. (2011). Age-related changes in an antioxidant defense system in elderly patients with essential hypertension compared with healthy controls. Redox Rep, 16 (2): 71- 77.

[46] Kasapoglu M., Ozben T. (2001). Alterations of antioxidant enzymes and oxidative stress markers in aging. Experimental Gerontology, 36: 209-220.

[47] Erden-Inal M., Sunal E., Kanbak G. (2002). Age-related changes in the glutathione redox system. Cell Biochemistry and Function, 20: 61- 66 .

[48] Quiroga G.B., Perez-Campo R., Lopez Torres M. (1990). Antioxidant defense and peroxidation in liver and brain of aged rats. Biochem. J, 272: 247-250.

[49] Dogru-Abbasoglu S., Tamer-Toptani S., Ugurnal B., KocakToker N., Aykac-Toker G. , Uysal M. (1997). Lipid peroxidation and antioxidant enzymes in livers and brains of aged rats. Mech. of Ageing and Dev, 98: 177- 180.

[50] Liu H. , Wang H. , Shenvi S. , Hagen T. M. , Liu R. M. (2004). Glutathione metabolism during aging and in Alzheimer disease. Annals of the New York Academy of Sciences, 1019: 346-349.

[51] Rebrin, I. , Sohal R. S. (2008). Pro-oxidant shift in glutathione redox state during aging. Advanced Drug Delivery Reviews, 60 $1545-1552$.

[52] Augustyniak A., Skrzydlewska E. (2004). Antioxidative abilities during aging. Postepy Hig Med Dosw, 58: 194- 201.

[53] Frayling T., Rafiq S., Murray, (2007). An interleukin-18 polymorphism is associated with reduced serum concentrations and better physical functioning in older people. J Gerontol ABiol Sci Med Sci, 62: 73- 78.

[54] Pavao M., Cordeiro C., Costa A. (2003). Comparison of whole-blood glutathione peroxidase activity, levels of serum selenium, and lipid peroxidation in subjects from the Fishing and Rural Communities of "Rabo de Peixe" village, San Miguel Island, the Azores' Archipelago, Portugal. Biol Trace Elem Res, 92: 27-40.

[55] Hiroshi Yamagami, Kazuo Kitagawa, Taku Hoshi, Shigetaka Furukado, Hidetaka Hougaku, Yoji Nagai, Masatsugu Hori, (2005). Associations of Serum IL-18 Levels With Carotid Intima-Media Thickness. Arteriosclerosis, Thrombosis, and Vascular Biology, 25: 1458- 1462.

[56] Das S., Yadav D., Narang R., Das N. (2002). Interrelationship between lipid peroxidation, ascorbic acid and superoxide dismutase in coronary artery disease. Curr Sci, 83(4): 488491.

[57] Busserolles J., Mazur A., Gueux E., Rock E., Rayssiguier Y. (2002). Metabolic syndrome in the rat: Females are protected against the pro-oxidant effect of a high sucrose diet. Exp Biol Med, 227: 837- 842. 
[58] Halliwell B., Gutteridge J.M.C. (2008). Free Radicals in Biology and Medicine, 4th ed. Oxford, Oxford University Press.

[59] Proteggente A. R., England T. G. , Rehman A., Rice-Evans C. A. , Halliwell B. (2002). Gender differences in steady-state levels of oxidative damage to DNA in healthy individuals. Free Radic. Res, 36: 157- 162.

[60] Sastre J. , Borras C. , Garcia-Sala D. , Lloret A. , Pallardo F. V., Vina J. (2002). Mitochondrial damage in aging and apoptosis. Ann. N.Y. Acad. Sci, 959: 448-451.

[61] Borras C., Sastre J., Garcia-Sala D., Lloret A., Pallardo F. V., Vina J. (2003). Mitochondria from females exhibit higher antioxidant geneexpression and lower oxidative damage than males. Free Radic. Biol. Med, 34: 546- 552.
[62] Bolzan A.D., Bianchi M.S., Bianchi N.O. (1997). Superoxide dismutase, catalase and glutathione peroxidase activities in human blood: influence of sex, age and cigarette smoking. Clin Chem, 30: 449- 454.

[63] Simic D.V., Mimic-Oka J., Pljesa-Ercegovac M. (2006). Byproducts of oxidative protein damage and antioxidant enzyme activities in plasma of patients with different degrees of essential hypertension. J Hum Hypertens, 20: 149- 155.

[64] Kedziora-Kornatowska K. , Czuczejko J., Pawluk H., Kornatowski T., Motyl J., Szadujkis-Szadurski L., Szewczyk-Golec K., Kedziora J. (2004). The markers of oxidative stress and activity of the antioxidant system in the blood of elderly patients with essential arterial hypertension. Cell Mol Biol Lett, 9: 635- 641. 